examined and compared treatment of Mâori (Indigenous) and nonMâori New Zealanders with colon cancer.

Methods: From the New Zealand Cancer Registry we identified a population-based cohort of 629 patients diagnosed with colon cancer between 1996 and 2003. We reviewed medical notes and compared surgical and oncology treatment in Mâori and non-Mâori patients. We adjusted treatment differences for tumour characteristics, patient comorbidity and treatment facility type.

Findings: Mâori and non-Mâori patients received similar rates of surgical resection although Mâori patients were less likely to have extensive lymph node clearance (relative risk (RR) $0.25,95 \%$ CI 0.13 to 0.50 for removal of 30 or more nodes) and were more likely to die in the post-operative period (RR 5.31, 95\% CI 1.54 to 18.32 for death following elective surgery). In patients with stage III disease Mâori were significantly less likely to receive chemotherapy (RR $0.69,95 \%$ CI 0.53 to 0.91 ) and more likely to experience delay of at least eight weeks to start chemotherapy (RR 1.98, 95\% CI 1.23 to 3.16). Treatment disparities were not accounted for by patient comorbidity or treatment facility type (public cancer centre, public non-cancer centre and private facility) although differences within facility types remain a potential explanatory factor.

Interpretation: Mâori and non-Mâori patients with colon cancer receive similar surgical treatment but Mâori are less likely to receive adjuvant chemotherapy and may experience a lower quality of care. Attention to health-system factors is needed to ensure equal access and quality of cancer treatment.

\section{SOCIOECONOMIC DIFFERENCES IN STATIN USE AFTER DEREGULATION OF SIMVASTATIN IN THE UK: THE WHITEHALL II PROSPECTIVE COHORT STUDY}

I Forde, T Chandola, MG Marmot, M Kivimaki. Department of Epidemiology and Public Health, University College London, London, UK

\section{doi:10.1136/jech.2009.096701n}

Objective: To examine socioeconomic differences in statin use after deregulation of simvastatin in adults with moderate or high risk of coronary heart disease.

Design: Prospective cohort study.

Setting: 20 civil service departments in London.

Participants: 3631 men and women (mean age 62.7 years) with moderate or high 10-year risk of CHD according to Framingham risk score.

Main Outcome Measures: Statin use, both prescribed and over the counter; recall of personal CHD risk.

Results: Based on medical screening, 2451 participants were at high $\mathrm{CHD}$ risk and 1180 at moderate risk. Of the high-risk participants, $54 \%$ reported using prescribed statin. This rate did not differ between employment grades (an index of socioeconomic position) after adjusting for age and sex; South Asian participants, however, were more likely to report using prescribed statin than White participants (odds ratio $1.73,95 \%$ CI 1.28 to 2.36 ). Three percent of high-risk participants reported using over the counter statin with participants from low (OR $0.11,95 \%$ CI 0.01 to 0.88 ) and middle (OR $0.54,95 \%$ CI 0.29 to 1.00 ) employment grades being less likely users than those from high employment grades. Among moderaterisk participants, $8 \%$ reported using over the counter statin; we found no variation by employment grade or ethnicity in this group. $37 \%$ of high-risk participants recalled their CHD risk. After adjusting for age, sex and cognitive function, South Asians were significantly less likely to recall than White participants (OR 0.65 , $95 \%$ CI 0.46 to 0.93 ) and middle (OR $0.74,95 \%$ CI 0.61 to 0.89 ) and low (OR $0.52,95 \%$ CI 0.37 to 0.74 ) employment grades participants less likely to recall than those from high employment grades.

Conclusion: Reported use of statin is considerably lower than need in all social groups although our data suggest that use of statin has largely remained socially equitable after recent changes in availability.
However, most high-risk participants are unaware of their risk, despite being informed of their risk by the study. Ethnic minorities and lower socioeconomic position groups, who are most at risk of heart disease, are significantly less likely to be aware. This is likely to impact on ability to participate in self-management and may partly explain poorer clinical outcomes.

\section{SOCIO-DEMOGRAPHIC PREDICTORS OF UPTAKE OF MMR IMIMUNISATION IN THE LIFEWAYS COHORT STUDY}

${ }^{1} \mathrm{~L}$ Jessop, ${ }^{1} \mathrm{CC}$ Kelleher, ${ }^{1} \mathrm{C}$ Murrin, ${ }^{1} \mathrm{~J}$ Lotya, ${ }^{1} \mathrm{D}$ O'Mahony, ${ }^{1} \mathrm{AT}$ Clarke, ${ }^{2}$ UB Fallon, ${ }^{3} \mathrm{H}$ Johnson, ${ }^{4} \mathrm{G}$ Bury, ${ }^{5} \mathrm{~A}$ Murphy, on behalf of the Lifeways Cohort Study Steering Group ${ }^{1}$ School of Public Health and Population Science, Woodview House, University College Dublin, Belfield, Dublin 4, Ireland; ${ }^{2}$ HSE Dublin Mid-Leinster Area Offices, Arden Road, Tullamore, Co. Offaly, Ireland; ${ }^{3}$ Health Information Unit, Health Intelligence, National Population Health Directorate, HSE, Dr Steevens Hospital, Steevens Lane, Dublin 8, Ireland; ${ }^{4}$ School of Medicine and Medical Science, The Coombe Healthcare Centre, Dublin 9, Ireland; ${ }^{5}$ Department of General Practice, National University of Ireland Galway, University Road, Galway, Ireland

doi:10.1136/jech.2009.0967010

Objectives: To investigate the uptake of the first dose measles, mumps and rubella (MMR) vaccine and factors associated with not receiving this vaccine.

Design: Cross-Generational Cohort Study with prospective linkage to primary care and hospital health records.

Setting: Urban and rural settings in Ireland recruited in 2001-2003 with five years follow-up.

Participants: 749 singleton children with immunisation information collected from the Health Service Executive (HSE). These are $70 \%$ of the original cohort who could be matched to HSE data.

Main Outcome Measures: Children immunised or not with the first dose of MMR by 2007.

Results: MMR uptake was $88.8 \%$ overall, with $91.6 \%$ in the East of Ireland (urban) and $83.8 \%$ in the West (rural). Odds ratios, adjusted for the other factors and the cluster affect of region, for the child not receiving $M M R$ were significant for the following independent factors; having a mother who had ever visited an alternative practitioner, OR 2.65 (1.76 to 3.98), a degree level educated mother OR 1.48 (1.07 to 2.04), an unmarried mother OR 1.97 (1.09 to 3.36), a mother who was a smoker OR 1.32 (1.04 to 1.69) or a lone parent OR 1.14 (1.13 to 1.15). Families that had 3 or more children in the household before the birth of the cohort child, OR 2.64 (1.42 to 4.91), had an income of less than $£ 300 /$ week OR 1.60 (1.35 to 1.91), who lived in the West of Ireland, OR 2.7 (2.47 to 2.96), or had a male child, OR 1.76 (1.74 to 1.78) were also significant factors. Conclusions: These novel prospective data confirm that in addition to factors associated with disadvantage, other health practices and beliefs may influence immunisation uptake. Furthermore, the determinants are heterogeneous. In light of the controversy around the MMR vaccine, attitudes of parents are highly relevant. This information can be used to design more focused parent information and immunisation services to target differing families whose children are at increased risk of measles infection. More work is needed to investigate parental beliefs as to why boys are less likely to receive the MMR. Specific interventions are vital if the target of $95 \%$ uptake of MMR is to be reached and measles is to be eradicated from the European region by 2010.

\section{FOOD POVERTY AND DIETARY QUALITY: IS THERE A RELATIONSHIP?}

'J Harrington, ' $\mathrm{J}$ Lutomski, ${ }^{2} \mathrm{M}$ Molcho, 'IJ Perry. 'Department of Epidemiology and Public Health, University College Cork, Cork, Ireland; '2Department of Health Promotion, NUI, Galway, Ireland

doi:10.1136/jech.2009.096701p

Background: Food poverty is intrinsically linked to inadequate income, poor dietary and lifestyle habits, and health inequalities, 
placing the "food poor" at higher risk of developing chronic diseases such as hypertension, diabetes and cardiovascular disease. Much of the evidence linking food poverty and poor diet is based on ecological level analysis. The Dietary Approaches to Stop Hypertension (DASH), a diet optimising dietary quality specifically limiting the intake salt, fats and sugars, has been shown experimentally to be associated with improved health outcomes, specifically hypertension and a DASH dietary score was developed which predicts a decreased risk of cardiovascular endpoints in observational studies.

Objective: Our objective was to examine the dietary habits of those who reported experiencing food poverty in relation to a DASH Score as an index of diet quality.

Methods: As part of the Survey of Lifestyle Attitudes and Nutrition (SLÁN)2007, 10364 adults aged 18 years and over were interviewed in their own homes (62\% response rate). Dietary habits were assed using a Food Frequency Questionnaire (FFO) ( $\mathrm{n}=9223$, response rate $89 \%$ ). A DASH dietary score (ranging from 9 to 42 ) was constructed using data from the FFO based on standardised methods. Higher DASH scores equated to healthier diets.

Results: Overall, $15 \%$ of the population could not always afford to buy the foods they wanted to, an indication of having experienced food poverty at least occasionally. The food poor had significantly worse dietary habits than the non-food poor: they were more likely to add salt to food, consume fried food frequently, and to have low DASH scores. Relative to those who had not experienced food poverty, the food poor had almost twice the odds of having a DASH score in the lowest two DASH Score quintiles (OR 1.7, 95\% CI 1.44 to 1.88 ).

Conclusion: Food poverty is negatively associated with dietary quality. Our results provide further evidence that those experiencing food poverty have staple diets that are high in salt, fats and sugars, thereby contributing to poor health outcomes among the most disadvantaged in our society.

\section{Mental health}

\section{EARLY MENARCHE IS ASSOCIATED WITH AN INCREASED RISK FOR DEPRESSIVE SYMPTOMS IN ADOLESCENT GIRLS IN A UK COHORT}

${ }^{1} \mathrm{C}$ Joinson, ${ }^{2} \mathrm{~J}$ Heron, ${ }^{1} \mathrm{R}$ Araya, ${ }^{1} \mathrm{G}$ Lewis. ${ }^{1}$ Department of Community Based Medicine, University of Bristol, Bristol, UK; ${ }^{2}$ Department of Social Medicine, University of Bristol, Bristol, UK

doi:10.1136/jech.2009.096701q

Objectives: To examine whether girls experiencing earlier menarche than their peers have higher levels of depressive symptoms in adolescence.

Design: Longitudinal cohort study.

Main Outcome Measures: Depressive symptoms were assessed using the Short Mood and Feelings Questionnaire (SMFQ). High levels of depressive symptoms were defined by scores on the SMFQ at or above 11. Age at onset of menarche was derived from regular questionnaires relating to pubertal development completed when the study children were 8 to 14 years old. An age at onset of menarche variable with three levels was derived (early: $<11.5$ years; on time: $11.5-13.4$ years; late: $>13.5$ years)

Participants: 2331 girls (age range $=12.6-15.2$ years; median $=13.8$ years) from a UK cohort study - The Avon Longitudinal Study of Parents and Children (ALSPAC).

Results: The unadjusted odds ratios for SMFQ score at or above 11 were 2.15 (95\% CI 1.43 to 3.24) for girls with an early onset of menarche and 1.62 (95\% CI 1.15 to 2.26 , global $\mathrm{p}$ value $=0.0008$ ) for girls with onset of menarche that was on time compared with girls with late onset of menarche. After adjusting for potential confounders of the association between onset of menarche and depressive symptoms (including socioeconomic disadvantage, absence of the biological father, body mass index and age at assessment of depressive symptoms), the odds ratios were 2.10 (1.37 to 3.21 ) for the early onset menarche group and 1.64 (1.16 to 2.32, global $p$ value $=0.0015$ ) for the "on time" onset group. There was an increase in strength of the association between onset of menarche and depressive symptoms from "on time" to "early onset" ( $p$ value for trend $=0.001$ ).

Conclusion: Early maturing girls are at increased risk for depressive symptoms in adolescence and could be targeted by school and family-based programmes aimed at early intervention and prevention. Adolescence is characterised by a marked rise in rates of depression in girls. This is of major concern to public health because depression has a chronic and recurrent course and is associated with impaired social functioning, low academic achievement, substance abuse and suicidal behaviour. Increased understanding of factors associated with the rise in rates of depressive symptoms in girls during adolescence is required to inform prevention programmes so that vulnerable individuals can be targeted.

\section{REGULATORY GUIDANCE ON PRESCRIBING SELECTIVE SEROTONIN REUPTAKE INHIBITOR ANTIDEPRESSANTS TO YOUNG PEOPLE: ECOLOGICAL STUDY OF EFFECTS ON INTERNATIONAL SUICIDE TRENDS}

B Wheeler, D Gunnell, C Metcalfe, R Martin. Department of Social Medicine, University of Bristol, Canynge Hall, 39 Whatley Road, Bristol, UK

doi:10.1136/jech.2009.096701r

Objective: In 2003 many international medicine regulatory agencies issued warnings concerning the prescribing of selective serotonin reuptake inhibitor (SSRI) antidepressants to young people, due to concerns that they increase risks of suicidal behaviour. A US study suggested that this action may have actually caused an increase in suicide rates due to under-treatment of depression, whilst a UK study did not find such an increase. This study investigates the population impact of globally significant regulatory action on youth suicide trends in a number of countries. Design: Ecological study.

Setting: 22 economically developed countries with relevant data in the WHO mortality database.

Populations: National populations aged 10 to 19 ears.

Main Outcome Measures: Annual suicide mortality rates (ICD10 codes X60-X84, Y10-Y34, Y87.0 and Y87.2, and equivalent ICD9 codes) from 1990 to the most recent date available for each country (2004-2006).

Results: Trends in suicide rates amongst young people varied substantially across the 22 countries. There was no clear, consistent change in trends following the regulatory action in 2003. Randomeffects Poisson regression models were used to model whether suicide rates post-2003 were higher or lower than expected, given country specific trends up to that date. Amongst 15-19-year-olds the rate ratio for the post-2003 period relative to preceding trends was 0.999 (95\% CI 0.971 to 1.028), and in 10-14-year-olds was 0.999 ( $95 \%$ CI 0.929 to 1.074). There was some evidence that trends differed in males and females. The rate ratios for 15-19-year-olds were 0.982 (95\% CI 0.950 to 1.015) amongst males and 1.081 (95\% CI 1.019 to 1.146) amongst females in the 15-19 age group, with a similar pattern amongst 10-14-year-olds, although with much greater uncertainty. Despite this average finding, post-2003 rates amongst 15-19-year-old females were lower than expected in a majority of countries (13 of 22).

Conclusions: There was no evidence of an overall effect of regulatory action to restrict prescribing of SSRIs to young people on suicide trends in these 22 countries, either favourable or unfavourable. 